\title{
Expressive Fused Deposition Modeling by Controlling Extruder Height and Extrusion Amount
}

\author{
Haruki Takahashi \\ Meiji University \\ Nakano, Tokyo, Japan \\ haruki@meiji.ac.jp
}

\author{
Homei Miyashita \\ Meiji University \\ Nakano, Tokyo, Japan \\ homei@homei.com
}

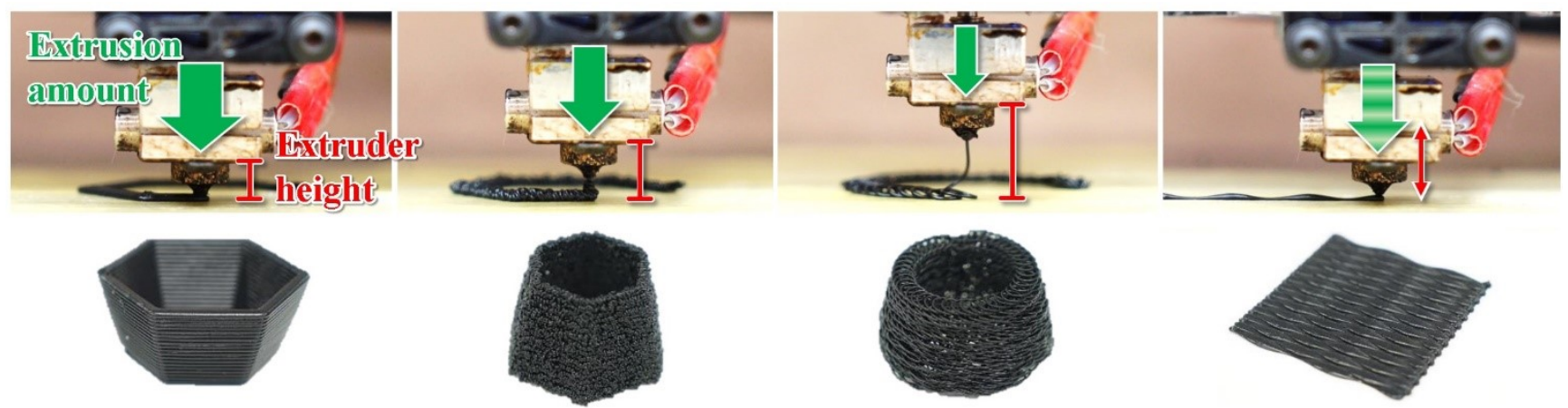

Figure 1. Extrusion state of our technique. We focus on (red) the height position of the extruder and (green arrow) the amount of extruded materials and explore the relationship between the combination of parameters and the behavior of extruded materials to extend the expression of the FDM 3D printer.

\begin{abstract}
Fused deposition modeling (FDM) 3D printers form objects by stacking layers having a linear structure. To print fine structures, an appropriate choice of parameters is necessary, or printing error occurs. On the other hand, the printing error is exploited as an expression technique. However, the relation between the printed structure and the parameters causing the printing error is unclear. In this paper, we focus on the height position of the extruder and the amount of extruded material, and explore the combination of these parameters to enhance the capability of FDM. By extending an equation that calculates the amount of material from the layer height, we investigate the behavior and structure of material extruded from various height positions. On the basis of experimental results, the printed structure is classified into six categories according to the structural feature. We describe these structural features and demonstrate examples with new inherent expressions for FDM.
\end{abstract}

\section{Author Keywords}

3D printing; fabrication; fused deposition modeling; expression; printing error.

\footnotetext{
Permission to make digital or hard copies of all or part of this work for personal or classroom use is granted without fee provided that copies are not made or distributed for profit or commercial advantage and that copies bear this notice and the full citation on the first page. Copyrights for components of this work owned by others than the author(s) must be honored. Abstracting with credit is permitted. To copy otherwise, or republish, to post on servers or to redistribute to lists, requires prior specific permission and/or a fee. Request permissions from Permissions@acm.org.

CHI 2017, May 06 - 11, 2017, Denver, CO, USA

Copyright is held by the owner/author(s).Publication rights licensed to ACM. ACM 978-1-4503-4655-9/17/05 \$15.00

DOI: http://dx.doi.org/10.1145/3025453.3025933
}

\section{ACM Classification Keywords}

H.5.m. Information interfaces and presentation (e.g., HCI): Miscellaneous;

\section{INTRODUCTION}

Advances in digital fabrication techniques such as threedimensional (3D) printers have enabled people to accurately and cheaply create 3D objects. In particular, fused deposition modeling (FDM) printers have become famous and are currently available in the market owing to their several advantages: ease of use, choice of materials, and low running cost [30]. Open-source 3D printers, especially those based on RepRap project [13], are specialized as highly customizable and are used by many manufacturers and researchers as the basis for commercial 3D printers or for experimental purposes.

FDM printers form an object by stacking thin layers, and each layer consists of a fine linear structure called "road" [5]. To print a high-quality object, we need to control and pay attention to each road by setting appropriate parameters or the $3 \mathrm{D}$ printers will fail to print stable objects and cause a printing error. On the other hand, this feature can be regarded as a freedom that allows users to access a layer in detail. On the basis of this feature and the printing error, a printing technique has been proposed (e.g., hair-like structure [16] and a scrubber [4]). The printing technique that exploits printing error requires parameters or ingenuity that is not commonly used in ordinary printing. These parameters are determined depending on each technique, and the relationship of the printed objects and an optimum value are unclear. Because many parameters are present to control a $3 \mathrm{D}$ printer and many of them causes printing error, 
investigating and discussing all combinations of parameters are impossible. However, 3D printers and its software are developed to prevent printing error. We have to understand this relationship or the usage of parameters or the technique that exploits printing error will be lost, and the capability and expression of FDM printers will become limited.

In this paper, we focus on the height position of the extruder and the amount of materials (Figure 1). We investigate the structure of a printed road and the behavior of extruded material by extending the range of parameters in this equation over the printing error, and we explore many combinations while changing their values during printing. Printed roads are classified into six categories according to their structural feature, which is denoted as distribution. Using our results, we implement design systems that enable users to design an object with a new expression. We print example objects to demonstrate new expressions for FDM. We extend the capability of FDM without using any new hardware and across various printing techniques.

\section{RELATED WORK}

\section{D printing error}

Printing error, where users cannot obtain the desired results, often occurs in FDM printers. To prevent a printing error, a troubleshooting guide for stable printing is provided (e.g., Simplify3D provides a troubleshooting guide [24]). Printing errors can be classified into two steps according to its types: printing process or printing quality.

First, a printer completely fails to print objects in the first place and cannot deposit roads on the platform or on previously printed roads. This error is caused by several problems such as lack of materials or setting error in the height position of the extruder, and users must prevent this error by referring to the guide. On the other hand, objects printed with error have quite stimulated and excited users in a different manner from the original objective. A few enthusiasts form a community to share pictures of the error and investigate the causes of trouble in 3D printing [8].

In addition, a technique that exploits the error as a novel printing technique has been proposed. Laput et al. presented a printing technique that can fabricate hair-like structures by exploiting the stringing (oozing) phenomena [16]. Kanada presented a method of 3D printing generative arts [14]. This technique consists of reflecting light control using the density of the filament. To change the density, the amount of materials is controlled, and such uneven extrusion is called over/under extrusion. Reiner et al. presented a method that can produce continuous tone imagery using an FDM 3D printer with two nozzles [21]. The contour of each layer is defined using sine pattern modulation and is overlaid over one another while shifting the modulation phase. This idea is considered as layer shifting. Blit It Inc. [4] provided a 3D printed scrubber called the O'Rourke Scrubber in its system. This scrubber is printed by extruding materials from a high position while randomly moving the extruder. Solid
Vibration [12] is a printing technique that can generate a Moiré pattern on the surface of objects. A platform is intensively and vertically vibrated, and the amount of materials being extruded is controlled by the change in the distance between the nozzle and the platform. Artist Lia developed software to produce custom Gcode to explore 3D printing as a sculpture technique [17]. By exploring the behavior of a 3D printer and its filament, the artist presented a new type of sculpture and methodologies.

The other error is related to printing quality such as surface roughness, dimensional accuracy, and strength. Functional parts require stricter requirements than the prototypes. For example, each part must be accurately and easily assembled after printing, and the final product must work as designed. Because many works related to $3 \mathrm{D}$ printers aim to address such printing quality problems, investigations of the relationship between the parameters and printed objects have been conducted, and optimization methods have been presented [28]. However, these works are performed on the assumption that printing error should be avoided. Thus, the range of parameters investigated in each study is restrictive.

Objects printed with a printing error often have a more complex and high-quality structure than the ordinary layerby-layer process. For example, thinner objects, which are impossible to print using the traditional method, are printed in 3D printed hair [16], and the surface roughness generated by the layered process (e.g., staircase effects [7]) is not noticeable. However, no investigation was conducted that considers a printing error as a new expression.

\section{Parameters for 3D printing}

The 3D printing process involves many variables, and 3D printers are controlled by setting several parameters to determine these variables. Agarwala et al. divided these variables into four categories (operation-, machine-, material-, and geometry-specific parameters) and reported that these parameters are interdependent [1]. When researchers investigate parameters, several parameters are used as variables to achieve a particular goal, and the other parameters are used as constant. For example, layer thickness significantly affects surface roughness $[2,3]$, and printing speed and paths affect the quality of layers and bonding of adjacent roads $[11,25]$. These parameter investigations are important not only for commercial 3D printers but also for open-source 3D printers [9, 15].

A part of the process is modeled as a numerical expression [27]. This approach is called process modeling and considered as the most significant research to understand the basic science behind the $3 \mathrm{D}$ printing process. Bellini conducted a comprehensive experiment on the FDM process and presented several models [5]. In addition, the spreading of extruded materials [6], deformation of objects printed using acrylonitrile butadiene styrene (ABS) materials [31], and pressure on the extruder (buckling phenomenon) [29] were investigated. Because these models are constructed in 
constrained environment, some problems are encountered in applying them to actual environment.

\section{D printing technique in $\mathrm{HCl}$}

Many human-computer interaction (HCI) researchers have presented a printing technique that can print objects using a unique structure or texture to achieve a new workflow and interaction.

Mueller et al. presented WirePrint [18] that prints objects using a wireframe mesh and allows users to quickly verify their design. Gannon et al. presented Tactum [10] that captures user skin-centric gestures for 3D modeling directly on the body. Torres et al. focused on the feel aesthetics of printed objects and proposed HapticPrint [26] that allows users to create a haptic design in passive $3 \mathrm{D}$ printed objects. To control the touch feeling, various textures are attached to the surface of objects. Schüller et al. focused on the surface of objects and proposed a framework to design an appearance that mimics surfaces (also known as bas relief) [22]. Cilllia [19] is a bottom-up printing pipeline to fully utilize the capability of high-resolution $3 \mathrm{D}$ printers, and a sophisticated hair-like structure is printed. This structure not only expands the expression of 3D printer but also enables users to design a passive actuator and a sensor. Highresolution $3 \mathrm{D}$ printers and a microstructure enable users to design deformable objects [23]. This structure is also called metamaterial and has attracted attention. These works provide an interaction technique through $3 \mathrm{D}$ printers and printed objects. To improve and understand the performance of 3D printers, a comprehensive study is needed.

\section{EXPRESSIVE FUSED DEPOSITION MODELING}

We extend the capability of the FDM printer to enable users to print new and expressive objects. Such printing techniques, which include the works we mentioned as related works, allow FDM printers to output complex objects that are difficult to print even by high-resolution 3D printers (e.g. stereolithography, selective laser sintering, and PolyJet). Moreover, these high-resolution 3D printers require a highresolution 3D model. Ease of use is not only a popular topic in the HCI field but also a strong point of an FDM printer. We allow users to easily express textures because our experiment is based on traditional parameter relationships. Because texture is generated during printing by the behavior of the materials, our technique does not require extra 3D modeling to design textures. Our technique only requires new parameters without any new hardware or 3D modeling skills, and it differentiates an FDM printer from other types of printers. We can provide a reason for choosing an FDM printer as a rapid prototyping tool.

Extending the capability of a 3D printer is a workflow design, which allows users to fabricate different and desirable objects, and it is an important topic in the field of digital fabrication in HCI. Within the context of HCI, a 3D printer can be considered as an output device such as a display. We provide a new perspective to a display (e.g., tactile display), whereas a high-resolution display is sought. Although our

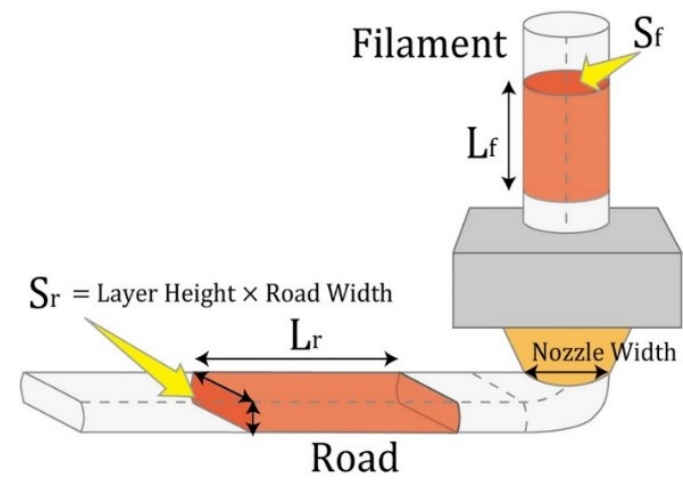

Figure 2. Schematic of the extrusion state.

approach differs from the mainstream, it is variable and belongs to the HCI field. We first describe the process of traditional FDM printers and how we extend the parameters.

\section{PROCESS OVERVIEW}

\section{Parameters in Gcode}

We use FDM 3D printers based on RepRap projects (opensource printer) whose printers can accept any Gcode. Gcode is a series of commands to control a $3 \mathrm{D}$ printer and is generated by converting a 3D model using slicer software. The following are sample codes:

$1 \quad$ G1 Z0.2

2 G1 X0 Y0 F3600

$3 \mathrm{G} 1 \mathrm{X} 10 \mathrm{Y} 10 \mathrm{E} 4 \mathrm{~F} 1800$

G1 is a command that controls the extruder and can accept several parameters. The first line means that the extruder vertically moves in the $Z$-axis direction (such movement is inserted at the start of each layer). The second and third lines mean that the extruder horizontally moves on the $X Y$-plane at a specific speed, and in the third line, the printer extrudes $4 \mathrm{~mm}$ of materials while moving to the end point $(10 \mathrm{~mm}, 10$ $\mathrm{mm})$. We focus on the parameter related to the height position of the extruder and the amount of materials, which are represented as parameters $Z[\mathrm{~mm}]$ and $E[\mathrm{~mm}]$ in Gcode, and control the printing process by changing these values.

These parameters are especially important for high-quality printing $[2,3]$, and the equation to calculate the amount of materials using several parameters, including the height position of the extruder, has already been defined. As the number of parameters increases, this relationship becomes more complex. Therefore, we prioritize these parameters and use other parameters as constant values.

\section{Calculation of material amount}

We illustrate the condition of the material being extruded from a nozzle (Figure 2). A linear material (filament) is fed into the heated nozzle; subsequently, the melted material is extruded. The extruded material takes a certain size (layer height and road width) and forms a road on the platform. The amount of material is calculated based on the relationship in 
which the volume of the extruded material (approximated in a cuboid shape) is equal to the volume of the material fed into the nozzle. Thus, by letting a cross-sectional area of a filament as $S_{f}\left[\mathrm{~mm}^{2}\right]$ and its length as $L_{f}[\mathrm{~mm}]$ and by letting a cross-sectional area of a printed road as $S_{r}\left[\mathrm{~mm}^{2}\right]$ and its length as $L_{r}[\mathrm{~mm}]$, the following relationship holds:

$$
S_{f} \times L_{f}=S_{r} \times L_{r} .
$$

Using Eq. (1), the length of material that should be fed $\left(L_{f}\right)$ to print the desired length of the road $\left(L_{r}\right)$ is calculated. $S_{f}$ and the width of the road are constant values determined by the diameter of the filament and the nozzle, respectively. The layer (road) height is determined by a corresponding parameter, which is given by the slicer software. Because parameter $E$ in $G l$ is equal to $L_{f}$, Eq. (1) can be rearranged as follows:

$$
E=\frac{\text { LayerHeight } \times \text { NozzleWidth } \times L_{r}}{S_{f}}
$$

In this paper, we refer to the layer height in Eq. (2) as the height position of the extruder. We conduct an experiment on the height position of the extruder and the amount of materials using the parameters derived from the extension of Eq. (2).

\section{EXTENSION OF EQ. (2)}

Equation (2) can be regarded as a linear function of the layer height when the length of the printed road $L_{r}$ is $1 \mathrm{~mm}$ (one unit of distance). Figure 3 shows the relationship between the height position of the extruder and the amount of materials. The bold line indicates the relationship of Eq. 2. We note that the relationship is calculated using a $1.75-\mathrm{mm}$ filament diameter and 0.4-mm nozzle width.

We consider the linear function to be in a polar coordinate form to explore the combination of various parameters based on this relationship. To express the function, we define two parameters, namely, $\theta\left[{ }^{\circ}\right]$ and $r[\mathrm{~mm}]$. First, Eq. 2, represented by the bold line, is expressed using $\theta=45^{\circ}$ to consider the parameter sets given by its relationship as a basis. To perform the calculation according to this basis, we adjust $\theta$ by multiplying the amount of materials by a correction value, which is the inverse of NozzleWidth $\times L_{r} / S_{f}$ in Eq. 2 (approximately 6.013 in this case). We introduce arbitrary values for $\theta$ and $r$ to calculate the height position of the extruder and the amount of materials as follows:

$$
\begin{gathered}
H=r \cos \theta \\
E=r \sin \theta \times \frac{\text { NozzleWidth }}{S_{f}} \times L_{r}
\end{gathered}
$$

where $H$ is the height position of the extruder [mm] and $E$ is the amount of extruded materials [mm]. For example, when the road is printed with a $0.3-\mathrm{mm}$ layer height, this height position of the extruder and the amount of materials are calculated using $\theta=45^{\circ}$ and $r=0.3 \times \sqrt{2}=0.4243 \mathrm{~mm}$.

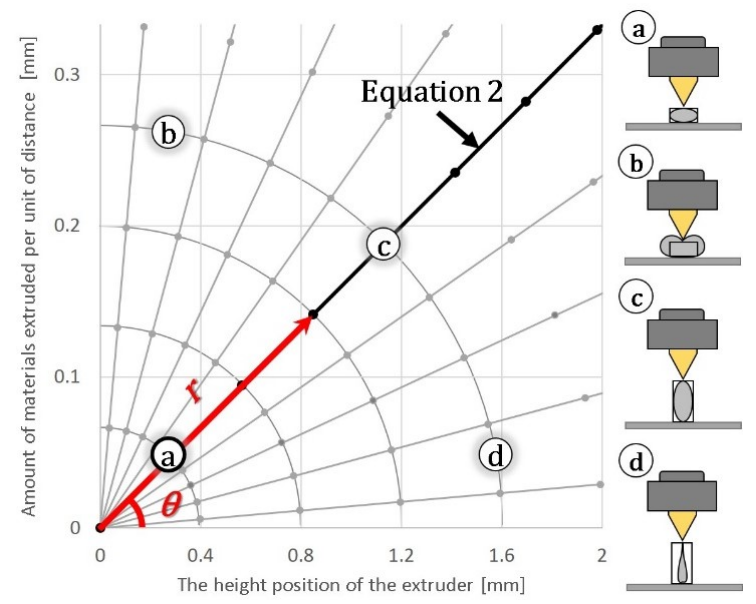

Figure 3. Relationship between the height position of the extruder and amount of materials: $0.4-\mathrm{mm}$ nozzle width and $1.75-\mathrm{mm}$ filament diameter. The traditional relationship is shown by a bold line. Our parameters $(r, \theta)$ and their relationship are shown by the red line. (a)-(d) Extrusion state of each parameter combination shown at the right side.

Using this relationship, we explore the parameter space while controlling the $\theta$ and $r$ values. Figure 3 shows the lines centered at a $45^{\circ}$ line at $10^{\circ}$ intervals and the concentric circles indicating the $r$ values.

The points from $a$ to $d$ in Figure 3 correspond to the figures at the right side, which show the approximate shape of the road and the state of extrusion of each parameter. Point $a$ is an ordinary parameter, and $3 \mathrm{D}$ printers can print a stable road around this point. In Point $b$, the amount of materials is larger than the height, and the roads may swell to enclose the nozzle or beyond the given parameter height. Although Point $c$ is set as a parameter based on Eq. (2), it indicates printing of a long lengthwise rectangular parallelepiped due to the constant nozzle diameter. According to the calibration guide ${ }^{1}$, best results are obtained when the layer height is $80 \%$ or less than the nozzle diameter and the road width is more than the nozzle diameter. However, results with excessive parameter values are unclear. Point $d$ is similar to Point $c$ but also lacks materials. In this case, the $3 \mathrm{D}$ printers may not print a road or fail to print at all. In the next section, we present the investigation of the behavior of the extruded materials and printed roads by actually printing various parameters.

\section{EXPERIMENT: PRINTING ROAD USING VARIOUS PARAMETERS}

\section{Design and procedure}

We conducted an experiment to explore the parameter space using Eqs. (3) and (4). We made the 3D printer print a road using various parameters. The length of the road along the $x$ axis was $200 \mathrm{~mm}, \theta$ was from $1^{\circ}$ to $90^{\circ}$ with an increment of $1^{\circ}$ (90 roads were printed), and $r$ was from 0.1 to $10.0 \mathrm{~mm}$, which continuously increased by $0.1 \mathrm{~mm}$ per printing of a 2.0-mm partial road. We used two materials made of ABS

\footnotetext{
${ }^{1}$ http://reprap.org/wiki/Triffid_Hunter's_Calibration_Guide
} 

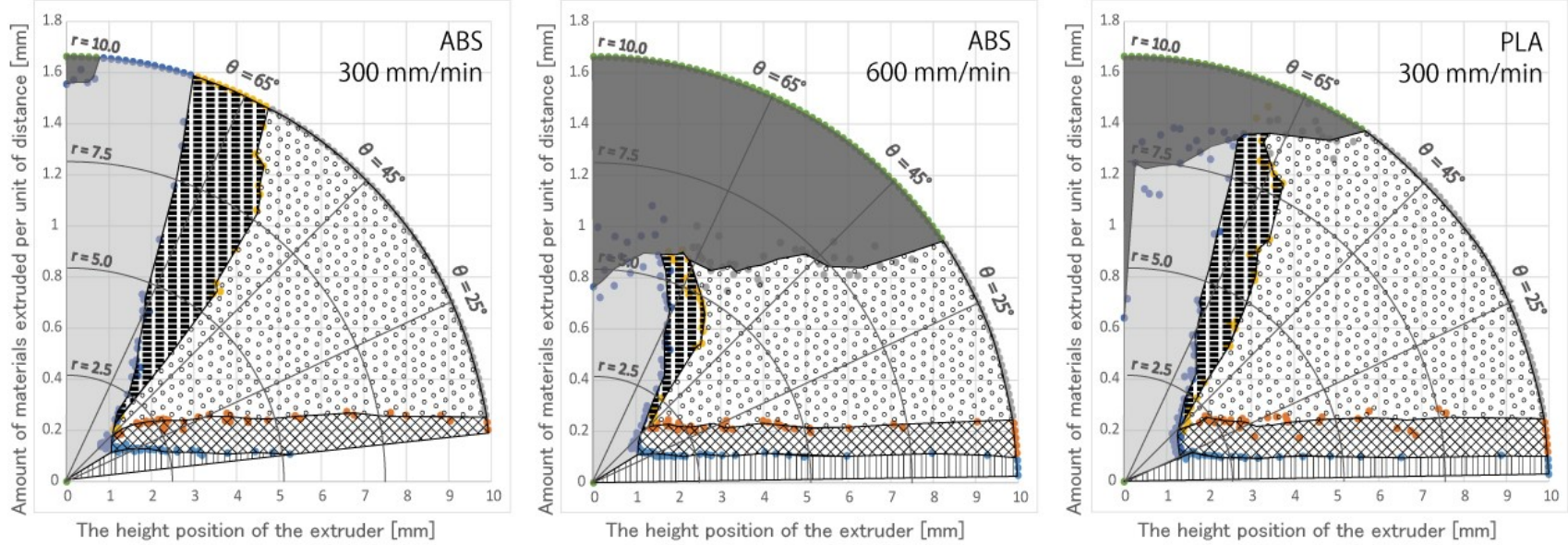

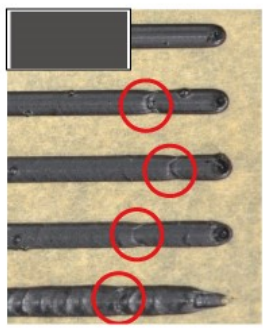

Stepping out of motor

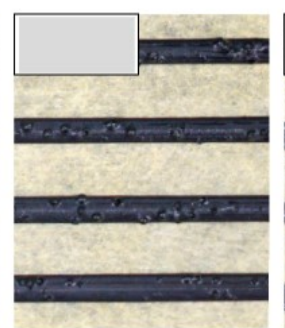

Stable road

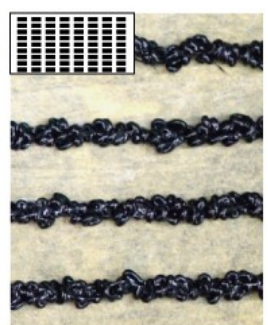

Zigzag road

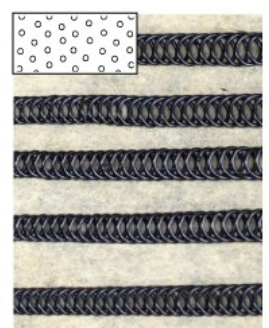

Coil-like road

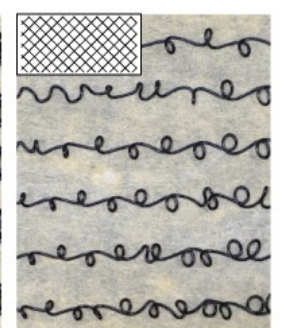

Frizzy hair-like road

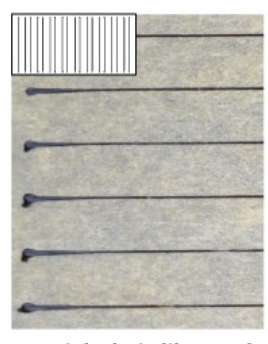

Straight hair-like road

Figure 4. (Top) Distribution of the printed structure. (Bottom) Printed objects classified into six categories.

and polylactic acid (PLA): MakerBot True Black ABS and Polymaker PolyMax PLA Black, respectively. We basically set the printing speed (feed rate) at $300 \mathrm{~mm} / \mathrm{min}$ in both materials, but we also set it at $600 \mathrm{~mm} / \mathrm{min}$ in the ABS to observe the difference in the printing speed.

To generate Gcode for the experiment, we implemented a system that can generate a road using specific parameters. Five roads generated with their respective $\theta$ values were placed side by side at an 8-mm interval, and we repeated the process to generate and print roads while changing the $\theta$ values. To minimize involuntary errors and the effect of oozing, we printed all roads according to the following procedures.

Slow extrusion $(6 \mathrm{~mm}$ at $10 \mathrm{~mm} / \mathrm{s}$ )

Quick retraction (3 $\mathrm{mm}$ at $30 \mathrm{~mm} / \mathrm{s}$ )

Three-second pause (cleaning off the nozzle)

Quick extrusion ( $3 \mathrm{~mm}$ at $30 \mathrm{~mm} / \mathrm{s}$ )

Printing a 200-mm road while increasing the $r$ value

Quick retraction $(3 \mathrm{~mm}$ at $30 \mathrm{~mm} / \mathrm{s}$ )

This process was also performed by the system and introduced into all roads as pre- and post-processing.

\section{Environment}

We used the FDM 3D printer Ninjabot NJB- $200^{2}$ with a 0.4mm-diameter nozzle and can extrude ABS and PLA. We

\footnotetext{
2 http://ninjabot.jp/product/fdm-200/

${ }^{3}$ http://www.repetier.com/
}

rewrote the setting of the maximum extrusion speed, which was written in a nonvolatile memory (EEPROM), because the printer firmware originally restricted the maximum extrusion speed to a small value. This value was used to set the maximum level and did not affect the amount of materials. We used Repetier-Host ${ }^{3}$ software that can edit and print Gcode to rewrite the above settings and manage the printing.

The temperature of the printer head and the built platform were 230 and $100{ }^{\circ} \mathrm{C}$ for ABS and 190 and $50^{\circ} \mathrm{C}$ for the PLA, respectively. To stabilize the printing, we applied a $50-\mathrm{mm}-$ wide washer tape on the built platform and a thin layer of paste. All roads were printed on this tape. The laboratory temperature was regulated to approximately $25{ }^{\circ} \mathrm{C}$, and the humidity was approximately $60 \%$.

\section{Results}

Figure 4 shows the classification of the results as a distribution. The printed roads are classified into six categories according to the structural feature (see Figure 4, bottom). This classification is based on the position measured using a digital caliper where the structure of the printed road was varied. We visually recognize such characteristic points. Figure 5 shows example roads printed at $\theta=60^{\circ}, 45^{\circ}$, and $20^{\circ}$.

\section{Structural feature of printed roads}

We describe and analyze the structural feature and material behavior in each category: stepping out of motor, stable road, zigzag road, coil-like road, frizzy hair-like road, and straight hair-like road. 


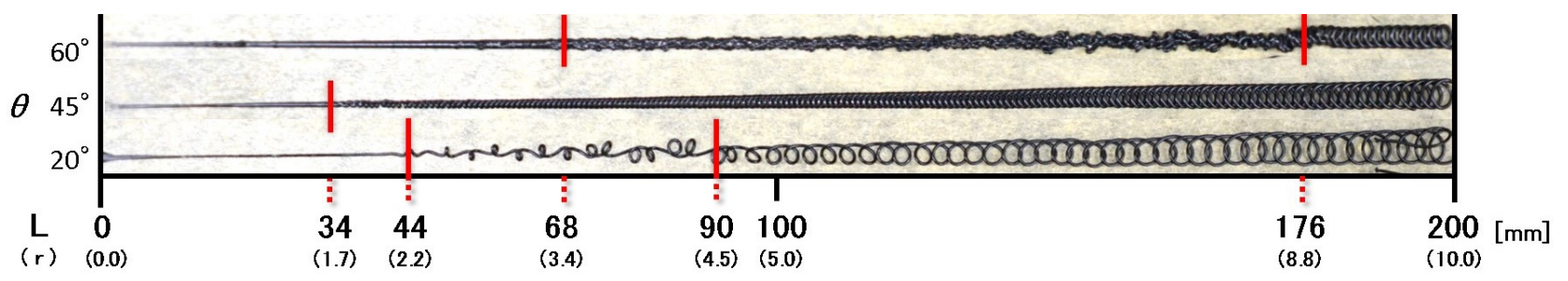

Figure 5. Variation in the road structure (ABS at $300 \mathrm{~mm} / \mathrm{min})$. Red lines indicate the change points of the structural feature.

\section{Stepping out of motor}

The 3D printer can stably extrude materials even if the parameters exceed their ordinary values. An exception to this performance is when the amount of materials is extremely large. In this case, the stepping motor, which consists of the extruder, frequently steps out, and the road becomes partially thin or disconnected fragments due to unstable extrusion. Printing speed also affects this phenomenon, and this area in the distribution becomes larger as the speed increases (see Figure 4; ABS at $600 \mathrm{~mm} / \mathrm{min}$ ). In PLA, although this area is also large, the cause could be due to the nozzle temperature. PLA was printed at $190^{\circ} \mathrm{C}$. By taking the average amount of materials when stepping out occurs, the limit of the extrusion speed can be estimated. The printer used in this experiment can extrude $\mathrm{ABS}$ of up to $11 \mathrm{~mm}^{3} / \mathrm{s}$.

\section{Stable road}

When stepping out does not occur, a road is printed at a stable state. We classify the road with traditional parameters into this category. The road becomes thicker as the amount of materials increases. However, some thicker roads with ABS have a rough surface. Figure 4 shows relatively thick roads. This roughness may be caused by changes in temperature and distortion of the road [5]. Materials that have just been extruded have high temperature and are deformed as they are gradually pushed up by other materials extruded later. A road part that cannot stand this deformation hardens and remains distorted or show traces of exploding air bubbles. In PLA, this distortion is not observed.

\section{Zigzag road}

As the height increases, materials are extruded with an uneven structure. We call this as a zigzag road. The road also becomes thicker and rougher as the amount of materials increases. This road cannot be printed with traditional parameters and obviously looks like a printing error. However, we focus on the appearance and rough texture, which is difficult to print even by high-resolution 3D printers.

\section{Coil-like road}

When $\theta$ is less than $60^{\circ}$, a coil-like road is printed. This category widely spreads out in terms of distribution, and this structure differs depending on the combination of parameters, e.g., a spiral with $\theta=20^{\circ}$ has a sparser structure (see Figure 5 ). This structure is printed by extruding materials from a high position similar to the zigzag road. This condition occurs because the parameter may have met several requirements to print this road. This road is not broken by the materials being extruded and can maintain its structure until it becomes stiff. In addition, the road is twisted in two directions (clockwise and counterclockwise). The direction of rotation is basically maintained during printing. However, entanglement in the middle of the road or reversal in the direction is seldom observed.

\section{Frizzy hair-like road}

As the amount of materials decreases, a coil-like road becomes a hair-like road. Distinguishing this road from the other will be described later. We classified this case as frizzy hair-like road. This road is created depending on the amount of materials because this category covers a rectangular area in every distribution (see Figure 4).

\section{Straight hair-like road}

Finally, a small amount of materials is extruded and pulled by the nozzle, and a straight hair-like road is printed. This condition can be considered to be the same as a structure printed in 3D printed hair [16]. When this road is printed, the whole road floats in midair. Thus, the starting point of the road must be fixed on the platform or the extruded materials are wiped out by the extruder movement. Moreover, when the printing speed is slow, the material tends to be burned out by the heat of the nozzle. The blank space, which is especially the lower part of $A B S$ at $300 \mathrm{~mm} / \mathrm{min}$, indicates this phenomenon, and we were not able to obtain a road with this parameter $\left(\theta<7^{\circ}\right)$. Although this phenomenon was already discussed in [16], we reproduce it using our approach.

\section{DESIGN SYSTEM}

We implement three design systems that enable users to utilize our results. The systems are implemented with vvvv, which is a visual programming language and renders graphical objects using DirectX, and we developed plugins with C\#. To implement the design system, additional parameters are needed beforehand to stack up or align each road (Figure 6). In the current system, users have to beforehand measure these properties according to each

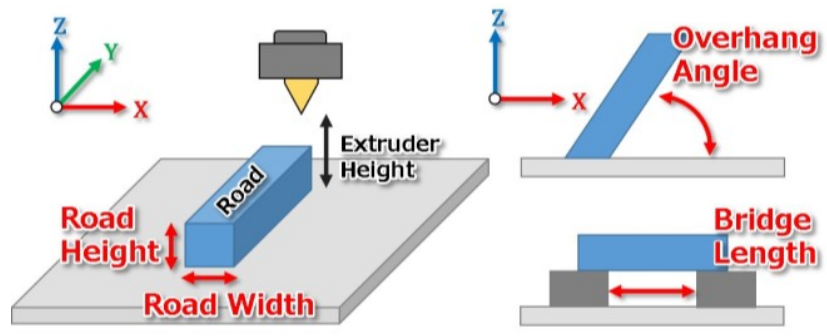

Figure 6. Additional parameters required to implement a design system.

\footnotetext{
${ }^{4}$ https://vvvv.org/
} 
combination of parameters using a digital caliper and input its measurement result to each design system. For example, the height position of the extruder for the first layer is obtained from Eq. (3), and all subsequent layers are sorted according to the road height, which is obtained by measurement. Thus, we focus on several combinations of parameters in the following section.

\section{\#1 Slicer using zigzag road}

First, we implement the slicer software that can be used in the same manner as a traditional one. This slicer analyze whether a support structure is needed or not and slice a 3D model into the contour and the inner structure according to the road height. If the support structure is needed, the slicer generates it around the 3D model. Overhang angle and bridge length are used to generate a support structure. Figure 7 shows the analysis process, a preview, and a printed result. To print the Stanford Bunny with a zigzag road, we used a parameter set $\left(\theta=65^{\circ}\right.$ and $\left.r=7.0 \mathrm{~mm}\right), \mathrm{ABS}$, and additional parameters measured before slicing.
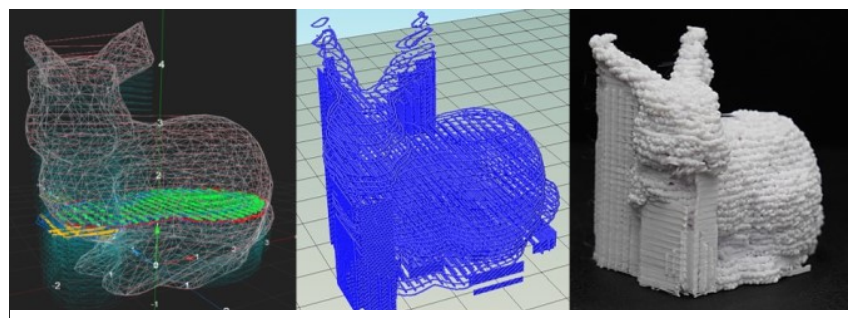

Figure 7. Slicer software with a zigzag road.

\section{\#2 Emboss processing}

Figure 8 shows the system for emboss processing. This system uses two parameters to generate bumps on the surface of printed objects; we intend to print a simple embossed wall in the current system. Users create and import a binary image, and the system then generates Gcode with different parameters according to its tone. To print the embossed object, we used two parameters (see Figure 8) and measured the road width of each parameter. Because these parameters have the same height, we can only change the width to express bumps.
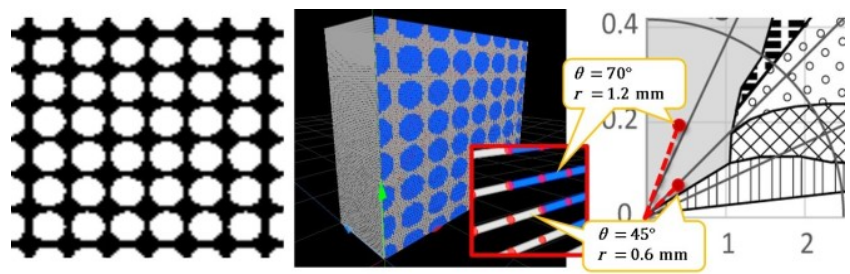

Figure 8. Design system for emboss processing (see Figure 10).

\section{\#3 Wavy sheets}

Wavy sheets also use two parameters to express the smooth surface. To generate wavy paths, this system generates numerous micro movements and assigns parameters to every path. Figure 9 shows the wavy paths divided into micro movements. The system requires two parameters (top and bottom) and complements the path between these according to the height position of the extruder.
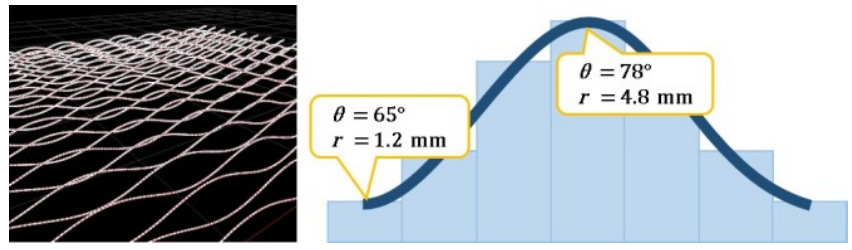

Figure 9. Design system for wavy sheets (see Figure 10).

\section{EXAMPLES}

We show 2D and 3D examples with various materials and parameters (Figure 10). A part of these examples is printed using the above design systems. Note that we adjusted the printing speed to prevent stepping out of motor and used the recommended temperature for each material.

Staircase effect represents an example constructed with thick roads. The staircase effect is one of the notable printing errors, which means that a stair is created on the surface of objects due to the thick layers. On the other hand, this roughness can provide a sense of stroking bellows, and we can easily control its condition using our method.

Weaver-nest-like objects are constructed by the zigzag road and have a quite rough surface. However, the whole structure is stable, and the printed object resembles a nest of a weaver

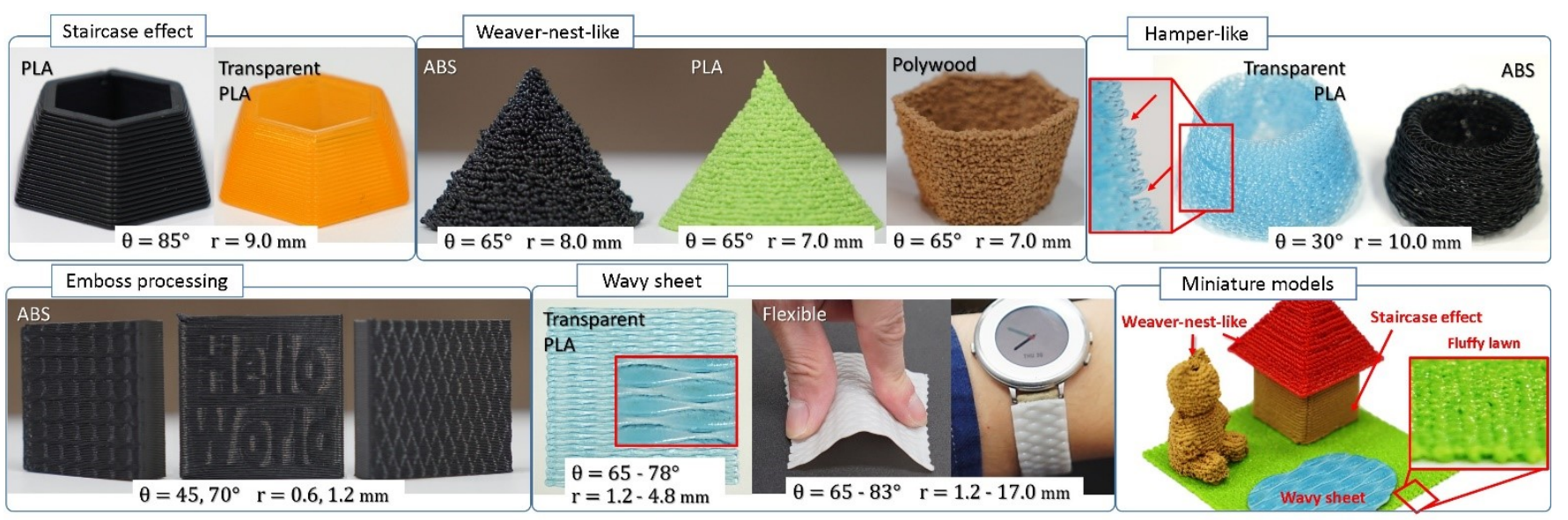

Figure 10. Example objects with new expressions. The materials and parameters used in each example are shown. 
bird. In PLA and Polywood, we decrease the $r$ value to obtain a zigzag road. When $r$ has the same value as that in ABS ( $r$ $=8.0 \mathrm{~mm}$ ), the printed road partially becomes a coil-like road.

Hamper-like objects are constructed by coil-like roads and have a flowery structure. Stacking this road is comparatively difficult. In particular, it becomes unstable around the start and end points of each layer, and the rotation direction of the coil varies in several layers.

Emboss processing is an expression using thickness control. We used the design system and two parameters to control the amount of materials with the same layer height. The overextruded material draws patterns and characters on the wall of the objects. Although this printing technique is not novel [14], we quantitatively show a method of setting the parameters as well as the relationship between the overextrusion and the parameters.

Wavy sheet is an object that is tactile and has a smooth pattern, and we print it using the design system that dynamically changes the parameters according to its shape. Using a flexible material and attaching it to a watch strap, we can change the sense similar to touching something that resembles an alligator hide. To print a larger bump, we also used an extremely large $r$ value $(r=17.0 \mathrm{~mm})$. Although this value exceeded the range in our experiment, the $3 \mathrm{D}$ printer was able to print the thicker part by reducing the printing speed to avoid stepping out of the motor. This printing technique can be applied to adaptive slicing [20], which controls the road thickness to minimize volumetric error.

By combining these expressions, we created miniature models and a diorama. In this example, we used our method to express all objects, which were a fluffy lawn, hair of a bear, a water surface, and a wall and roof of a house. An expressive printing technique enables users to print the delicate features.

\section{DISCUSSION}

Using the distribution, users can control the structure, and the design systems contribute to the utilization of our results. We received favorable responses from people who physically handled our examples; they described that the printed objects have a handmade feeling. We believe this comment implies the important aspect of personal fabrication.

However, an obscure area exists around the boundary of every category because each structure can be affected by the preceding and immediately following structures. Therefore, when the parameter is extremely varied or curved and polygonal roads are printed, the printed structure may be different from that in our result. In the experiment, we used several parameters as constant, and the effect of the environment is still significant. We applied the same parameters to other printers: Genkei Atom ${ }^{5}$ and Bonsai Lab. BS01+6 (Figure 11). When we printed examples using different printers and materials, we had to adjust $\theta, r$, and the

\footnotetext{
5 http:/genkei.jp/

${ }^{6}$ http://www.bonsailab.asia/
}

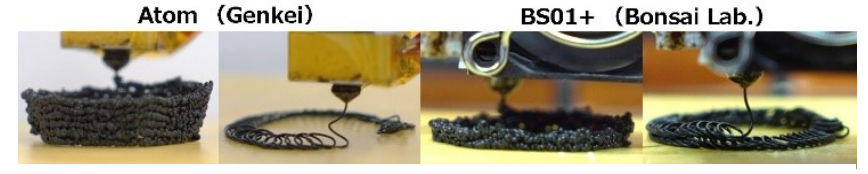

Figure 11. Application to other 3D printers.

printing speed once or twice. On the other hand, even if such constant is varied, the distribution of the parameters can be effectively utilized. For example, we reduced the printing speed to prevent stepping out in accordance with the distribution. Moreover, we could create the distribution of other materials in the same manner. If users want to control the printed structure in detail in their own environment, they need to clarify the features of the environment by conducting a similar experiment.

In our future work, we will investigate and provide the requirements to correctly print each structure. Although we classified the structures into six categories, each structure has different thickness and texture depending on the combination of parameters. For example, the stable road becomes thicker as the amount of materials increases. Some roads require a support structure similar to that of traditional printing to print in midair. On the other hand, several roads constructed with a complex structure (e.g., coil-like road) are difficult to print even with the support structure. We will also sensitively evaluate the objects printed with expressive FDM. Wang et al. conducted a user study to assess their objects in terms of both haptic and visual quality [32].

We provided the understanding related to the parameters, and it can open up a new interaction between human and 3D printers. We intentionally used descriptive and metaphorical words because we believe these are warranted by our result. Moreover, these can be used for designing textures (e.g., using natural language as input). Our work contributes to the development of the digital fabrication field in HCI.

\section{CONCLUSION}

In this work, we focused on the height position of the extruder and the amount of materials to extend the expression of FDM. We presented equations to explore the parameter space and conducted an experiment to investigate the combination of the parameters. The printed roads were classified into six categories according to the structural feature and were shown as a distribution of parameters. We implemented the design systems and printed example objects with our result to demonstrate new expressions.

Although many researchers and manufacturers pursue highquality printing using high-resolution and expensive printers, true high-quality objects are results that should satisfy the needs of users. To achieve such objects, users need to provide various expressions. We exploited even the printing errors to extend the capability of FDM 3D printers and allowed users to express the texture, surface condition, and appearance using a 3D printer.

\section{ACKNOWLEDGEMENTS}

This work was supported by COI, JST. 


\section{REFERENCES}

1. Mukesh K. Agarwala, Vikram R. Jamalabad, Noshir A. Langrana, Ahmad Safari, Philip J. Whalen, and Stephen C. Danforth. 1996. Structural quality of parts processed by fused deposition. Rapid Prototyping Journal, Vol. 2, No. 4, 4-19. http://dx.doi.org/10.1108/13552549610732034

2. Daekeon Ahn, Jin-Hwe Kweon, Soonman Kwon, Jungil Song, and Seokhee Lee. 2009. Representation of surface roughness in fused deposition modeling. Journal of Materials Processing Technology, Vol. 209, No. 15-16, 5593-5600. http://dx.doi.org/10.1016/j.jmatprotec.2009.05.016

3. R. Anitha, S. Arunachalam, and P. Radhakrishnan. 2001. Critical parameters influencing the quality of prototypes in fused deposition modelling. Journal of Materials Processing Technology, Vol. 118, No. 1-3, 385-388.

http://dx.doi.org/10.1016/S0924-0136(01)00980-3

4. Blit it.

https://blitit.com/

(Retrieved January 6, 2017)

5. Anna Bellini. 2002. Fused deposition of ceramics: a comprehensive experimental, analytical and computational study of material behavior, fabrication process and equipment design. Ph.D. dissertation, Drexel University, Philadelphia, PA.

http://hdl.handle.net/1860/22

6. Robert S. Crockett and Paul D. Calvert. 1996. The liquid-to-solid transition in stereodeposition techniques. In Proceedings of Solid Freeform Fabrication Symposium, 257-264.

7. André Dolenc and Ismo Mäkelä. 1994. Slicing procedures for layered manufacturing techniques. Computer-Aided Design, Vol. 26, No. 2, 119-126. http://dx.doi.org/10.1016/0010-4485(94)90032-9

8. Epic 3D printing fail.

http://epic3dprintingfail.tumblr.com/ (Retrieved January 6, 2017)

9. L.M. Galantucci, I. Bodi, J. Kacani, and F. Lavecchia. 2015. Analysis of dimensional performance for a 3D open-source printer based on fused deposition modeling technique, Procedia CIRP, Vol. 28, 82-87. http://dx.doi.org/10.1016/j.procir.2015.04.014

10. Madeline Gannon, Tovi Grossman, and George Fitzmaurice. 2015. Tactum: a skin-centric approach to digital design and fabrication. In Proceedings of the SIGCHI Conference on Human Factors in Computing Systems (CHI '15), 1779-1788.

http://dx.doi.org/10.1145/2702123.2702581

11. Wenbiao Han, Mohsen A. Jafari, Stephen C. Danforth, and Ahmad Safari. 2002. Tool path-based deposition planning in fused deposition processes. Journal of
Manufacturing Science and Engineering, Vol. 124, No. 2, 462-472.

http://dx.doi.org/10.1115/1.1455026

12. Olivier van Herpt. Solid Vibrations.

http://oliviervanherpt.com/solid-vibrations/

(Retrieved January 6, 2017)

13. Rhys Jones, Patrick Haufe, Edward Sells, Pejman Iravani, Vik Olliver, Chris Palmer, and Adrian Bowyer. 2011. RepRap - the replicating rapid prototype. Robotica, Vol. 29, No. 1, 177-191. http://dx.doi.org/10.1017/S026357471000069X

14. Yasushi Kanada. 2016. 3D printing of generative art using the assembly and deformation of directionspecified parts. Rapid Prototyping Journal, Vol. 22, No. 4, 636-644.

http://dx.doi.org/10.1108/RPJ-01-2015-0009

15. Antonio Lanzotti, Marzio Grasso, Gabriele Staiano, and Massimo Martorelli. 2015. The impact of process parameters on mechanical properties of parts fabricated in PLA with an open-source 3-D printer. Rapid Prototyping Journal, Vol. 21, No. 5, 604-617. http://dx.doi.org/10.1108/RPJ-09-2014-0135

16. Gierad Laput, Xiang 'Anthony' Chen, and Chris Harrison. 2015. 3D printed hair: fused deposition modeling of soft strands, fibers and bristles. In Proceedings of the ACM Symposium on User Interface Software and Technology (UIST '15), 593-597. http://dx.doi.org/10.1145/2807442.2807484

17. LIA. Filament sculptures. http://www.liaworks.com/ (Retrieved January 6, 2017)

18. Stefanie Mueller, Sangha Im, Serafima Gurevich, Alexander Teibrich, Lisa Pfisterer, François Guimbretière, and Patrick Baudisch. 2014. WirePrint: 3D printed previews for fast prototyping. In Proceedings of the ACM Symposium on User Interface Software and Technology (UIST '14), 273-280. http://dx.doi.org/10.1145/2642918.2647359

19. Jifei Ou, Gershon Dublon, Chin-Yi Cheng, Felix Heibeck, Karl Willis, and Hiroshi Ishii. 2016. Cilllia: 3D printed micro-pillar structures for surface texture, actuation and sensing. In Proceedings of the SIGCHI Conference on Human Factors in Computing Systems (CHI '16), 5753-5764. http://dx.doi.org/10.1145/2858036.2858257

20. Pulak Mohan Pandey, N. Venkata Reddy, and Sanjay G. Dhande. 2003. Slicing procedures in layered manufacturing: a review. Rapid Prototyping Journal, Vol. 9, No. 5, 274-288.

http://dx.doi.org/10.1108/13552540310502185

21. Tim Reiner, Nathan Carr, Radomír Měch, Ondřej Št'ava, Carsten Dachsbacher, and Gavin Miller. 2014. Dual-color mixing for fused deposition modeling 
printers. Computer Graphics Forum. Vol. 33, No. 2, 479-486.

http://dx.doi.org/10.1111/cgf.12319

22. Christian Schüller, Daniele Panozzo, and Olga Sorkine-Hornung. 2014. Appearance-mimicking surfaces. ACM Transactions on Graphics, Vol. 33, No. 6, Article 216.

http://dx.doi.org/10.1145/2661229.2661267

23. Christian Schumacher, Bernd Bickel, Jan Rys, Steve Marschner, Chiara Daraio, and Markus Gross. 2015. Microstructures to control elasticity in 3D printing. ACM Transactions on Graphics, Vol. 34, No. 4, Article 136.

http://dx.doi.org/10.1145/2766926

24. Simplify3D. Print Quality Troubleshooting Guide. https://www.simplify3d.com/support/print-qualitytroubleshooting/ (Retrieved January 6, 2017)

25. Q. Sun, G. M. Rizvi, C. T. Bellehumeur, and P. Gu. 2008. Effect of processing conditions on the bonding quality of FDM polymer filaments. Rapid Prototyping Journal, Vol. 14, No. 2, 72-80. http://dx.doi.org/10.1108/13552540810862028

26. Cesar Torres, Tim Campbell, Neil Kumar, and Eric Paulos. 2015. HapticPrint: Designing Feel Aesthetics for Digital Fabrication. In Proceedings of the ACM Symposium on User Interface Software and Technology (UIST '15), 583-591. http://dx.doi.org/10.1145/2807442.2807492

27. Brian N. Turner, Robert Strong, and Scott A. Gold. 2014. A review of melt extrusion additive manufacturing processes: I. Process design and modeling. Rapid Prototyping Journal, Vol. 20, No. 3, 192-204.

http://dx.doi.org/10.1108/RPJ-01-2013-0012

28. Brian N. Turner and Scott A. Gold. 2015. A review of melt extrusion additive manufacturing processes: II. Materials, dimensional accuracy, and surface roughness. Rapid Prototyping Journal, Vol. 21, No. 3, 250-261.

http://dx.doi.org/10.1108/RPJ-02-2013-0017

29. N. Venkataraman, S. Rangarajan, M. J. Matthewson, B. Harper, A. Safari, S. C. Danforth, G. Wu, N. Langrana, S. Guceri, and A. Yardimci. 2000. Feedstock material property - process relationships in fused deposition of ceramics (FDC). Rapid Prototyping Journal, Vol. 6, No. 4, 244-253.

http://dx.doi.org/10.1108/13552540010373344

30. W. Walters. 1992. Rapid prototyping using FDM: a fast, precise, safe technology. In Proceedings of Solid Freeform Fabrication Symposium, 301-308.

31. Tian-Ming Wang, Jun-Tong Xi, and Ye Jin. 2007. A model research for prototype warp deformation in the FDM process. The International Journal of Advanced Manufacturing Technology, Vol. 33, No. 11, 10871096. http://dx.doi.org/10.1007/s00170-006-0556-9

32. Weiming Wang, Cédric Zanni, and Leif Kobbelt. 2016. Improved Surface Quality in 3D Printing by Optimizing the Printing Direction. Computer Graphics Forum, Vol. 35, No. 2, 59-70. http://dx.doi.org/10.1111/cgf.12811 